\title{
Improving the Physical Activity of Breast Cancer Survivors Through Fitness Trackers
}

\author{
$\mathrm{C} \mathrm{LYNCH}^{\mathrm{a}, \mathrm{b}, 1}, \mathrm{~S}_{\mathrm{BIRD}}{ }^{\mathrm{a}}, \mathrm{F}$ BARNETT ${ }^{\mathrm{b}}, \mathrm{N} \mathrm{LYTHGO}^{\mathrm{a}}$ and I SELVA-RAJ ${ }^{\mathrm{a}}$ \\ ${ }^{a}$ Exercise Science, School of Health and Biomedical Sciences, \\ RMIT University, Bundoora, Victoria 3083, Australia \\ ${ }^{b}$ The Northern Health, Melbourne, Australia
}

\begin{abstract}
Introduction. Increasing physical activity among posttreatment breast cancer survivors is essential, as greater physical activity reduces the relative risk of cancerspecific mortality. This trial examines how a fitness tracker-based intervention changes the physical activity behaviour of inactive posttreatment breast cancer survivors. Methods. Seventeen physically inactive posttreatment breast cancer survivors participated in a randomised cross-over controlled trial. Participants underwent a 12-week intervention of a fitness tracker combined with a behavioural counselling and goal-setting session and 12 weeks of normal activity (control). The primary outcome was the change in physical activity assessed by accelerometry over seven days. Results. The intervention achieved a mean increase of $4.5 \mathrm{~min} /$ day of moderate-vigorous physical activity, representative of a small-moderate effect $(\mathrm{d}=$ $0.34)$. Changes in time spent as a proportion of the day in light physical activity ($8.3 \%)$ and in sedentary behaviour $(7.9 \%)$, were both significantly different to baseline $(\mathrm{t}(16)=3.522, \mathrm{p}<0.01 ; \mathrm{t}(16)=-3.162, \mathrm{p}<0.01)$. Conclusion. Interindividual differences in the change of patterns of physical activity behaviour suggest that only for some, fitness trackers can achieve a change in the level of moderate-vigorous physical activity.
\end{abstract}

Keywords. physical activity; fitness tracker; accelerometer; cancer survivors

\section{Introduction}

Physically inactive breast cancer survivors have an increased risk of cancer recurrence, cancer-specific and all-cause mortality. However, numerous observational studies show that being more physically active reduces that risk.[1] Furthermore, increasing physical activity (PA) among cancer survivors is an effective behavioural strategy for attenuating a decline in physical functioning, enhancing the health-related quality of life, and mitigating cancer-related fatigue.[2] Levels of PA among cancer survivors are lower than the population at large. Seventy to eighty percent of cancer survivors are insufficient in PA.[3] Many breast cancer survivors fail to achieve the recommended minimum of 150 min/week of moderate-intensity PA.[4] Therefore, there is a need to increase PA among breast cancer survivors.

The fitness tracker is an emerging and accessible technology that may facilitate behavioural change in PA. Fitness trackers and associated applications have the potential to change PA in cancer survivorship,[5] because they inherently contain behaviour-

${ }^{1}$ Corresponding Author, Chris Lynch, Exercise Science, School of Health and Biomedical Sciences, RMIT University, Bundoora, Victoria 3083, Australia; E-mail: chris.lynch@nj.org.au. 
change techniques, such as goal setting, self-monitoring of behaviour, prompts/cues, and social support.[6] Interventions for PA that have included a fitness tracker have shown an effect in changing PA behaviour; however, for step-count, the effect is only slightly greater to that of non-fitness tracker interventions.[7] Among cancer survivors, interventions using a fitness tracker have shown a positive effect in changing PA behaviour.[8] with trials underway in posttreatment breast cancer survivors.[9] However, with limited evidence, it is unclear to what extent the use of fitness tracker can change the PA behaviour of inactive breast cancer survivors.

The primary aim of this trial is to determine the effect of a 12 -week intervention using a fitness tracker, combined with a behavioural counselling and goal-setting session, to change the PA behaviour of inactive posttreatment breast cancer survivors.

\section{Methods}

\subsection{Participants}

Seventeen participants were recruited from an oncology outpatient clinic of a major hospital within metropolitan Melbourne, Australia. The trial was approved by the Austin Health Human Research Ethics Committee (LNR/17/Austin/338). Medical oncology staff identified potential participants during outpatient clinics. Participants identified by medical staff or who had expressed interest in the trial were sent an 'invitation' letter. Following a response to the letter, a brief telephone-delivered screening questionnaire was used to check eligibility.

All participants had to be aged $\geq 18$ years, diagnosed with breast cancer (stages IIII), and have completed primary and adjuvant treatments (hormone therapy excepted) within the last five years. Participants were physically inactive (engaging in $\leq 150$ minutes of moderate-intensity PA/week) with no contraindications for PA. Standard screening procedures assessed contraindications for PA.[10] Participants were required to have daily access to a smartphone, mobile device or personal computer. If eligible, the participant was invited to attend an initial trial visit.

\subsection{Measures}

During the initial trial visit and after giving written informed consent, participants completed a questionnaire recording breast cancer stage at diagnosis and treatments received. Socio-demographic and health characteristics were also recorded. Body mass index $\left(\mathrm{kg} / \mathrm{m}^{2}\right)$ was estimated from self-reported body mass $(\mathrm{kg})$ and height $(\mathrm{cm})$ using the standard $\mathrm{kg} / \mathrm{m}^{2}$ equation. Participants were instructed how to complete a baseline objective assessment of PA by accelerometry, to be conducted over the subsequent seven days.

Participants were provided with a tri-axial accelerometer (Actigraph GT 3X+; Actigraph, Pensacola, FL) and instructed to wear it on an elasticised belt over the nondominant hip for seven consecutive days, during all waking hours except during showering and aquatic activities. Written instructions on how to wear the accelerometer and a diary to record wear and non-wear time across the seven-day assessment period were provided. The purpose of the diary was to cross-check data and to refer to if unusual accelerometry patterns were noted. Accelerometry is a feasible method to assess PA in free-living cancer survivors[11] and valid for assessing moderate-intensity PA.[12] 
Accelerometer data were collected in one-minute epochs. On return of the accelerometer, these data were downloaded as activity counts. Activity counts represent raw accelerations summed over the one-minute epoch. These data were processed by the ActiLife software package (Version 6.12.1; Actigraph, Pensacola, FL). Data retained for analyses met a wear-time validation criterion of $\geq 10$ hours of wear-time for a minimum of three valid days, with an interruption period of 60 minutes. Each minute of wear-time was classified into PA intensities (counts/min) according to commonly accepted activity count cut-points.[13]

For each valid day, the number of wear-time minutes classified as sedentary, light-, moderate-, and vigorous-intensity PA was taken as the estimate of time spent in these activities. The number of minutes with intensity counts $>100$ was taken as an estimate of the total time spent active. Counts were summed over wear-minutes to obtain total valid counts for the reporting day. Minutes in each category were divided by wear-time to estimate proportions of the day spent in each behaviour. Daily estimates of the proportion of time spent sedentary and in each classification of PA were averaged across all valid days per participant to estimate the mean proportion of time spent in each behaviour.

\subsection{Intervention}

The trial was a cross-over trial design. Seven days after the initial trial visit, participants attended a second visit and a Web-based random number generator was used to assign participants initially into either the primary intervention $(n=10)$ or normal activity $(n=7)$ group. On completion of 12 weeks of primary intervention or normal activity (control) and after a seven-day washout period, participant assignment was reversed. The nature of the intervention meant the blinding of participants, and the blinding of the trial team to participant allocation was not feasible.

At the second trial visit, accelerometer data were downloaded, and a report of baseline PA from the seven-day assessment period was provided to all participants. A motivational interviewing approach guided behavioural feedback and goal setting. All participants were asked to think and record how they could increase their PA and set goals using the report of baseline PA.

Participants undertaking the intervention were provided with and trained in the setup and use of a fitness tracker, a Garmin Vivofit2. The selection of this tracker was based on earlier qualitative work.[14] Participants were shown typical examples of PA data provided by the fitness tracker and application. Participants were requested to wear and use the tracker for 12 weeks and were encouraged to regularly upload and access their PA data via the application. Trial team members were not able to access participantuploaded data.

\subsection{Statistical Analyses}

All statistical analyses were conducted using SPSS 26.0 statistical software program (SPSS Inc, Chicago, IL) and performed on the conclusion of the trial. Trial team members conducting statistical analyses were not blinded to participant allocation.

Numerical coding was used for categorical variables. The mean and standard deviation for all continuous variables were calculated. Normality of continuous variables was assessed by Kolmogorov-Smirnov test[15] with a visual inspection of histograms, Q-Q, and box plots. Significance for all statistical analyses was set at $p<0.05$. All analyses were unadjusted. 
A paired-samples $t$-test assessed the effect of the intervention on the primary outcome of PA. Cohen's $d$ calculated effect size statistics and the guideline proposed by Cohen (1988) was used for their interpretation.[16]

\section{Results}

At baseline, mean moderate-vigorous physical activity (MVPA) was $13.2( \pm 9.9) \mathrm{min} /$ day, representing $2 \%( \pm 1.5)$ of the day spent in this classification of PA. Participants spent the greatest proportion of the day in sedentary behaviour $(57.6 \%, \pm 9.9)$ and lightintensity PA $(40.4 \%, \pm 9.9)$.

Table 1. Participant characteristics, breast cancer stage at diagnosis, and treatments received.

\begin{tabular}{|c|c|c|c|c|}
\hline Variable $(n=17)$ & & & & \\
\hline \multirow[t]{2}{*}{ Age } & $(M, S D)$ & $<44 \quad(\%, n)$ & $\begin{array}{c}45 \text { to }<60 \quad(\%, \\
n)\end{array}$ & $(\%, n)$ \\
\hline & $49.3(9.4)$ & $35.3(6)$ & $53.0(9)$ & $11.7(2)$ \\
\hline \multirow[t]{2}{*}{ BMI kg/m ${ }^{2}$} & & $<25 \quad(\%, n)$ & $\begin{array}{c}25 \text { to }<30 \quad(\%, \\
n)\end{array}$ & $(\%, n)$ \\
\hline & $29.3(6.0)$ & $23.5(4)$ & $29.4(5)$ & $47.1(8)$ \\
\hline $\begin{array}{l}\text { Months since } \\
\text { completion of } \\
\text { primary treatment }\end{array}$ & $21.5(23.5)$ & & & \\
\hline \multirow[t]{2}{*}{$\begin{array}{l}\text { Primary disease stage } \\
\text { at the time of } \\
\text { diagnosis }\end{array}$} & & $\begin{array}{c}\text { Stage I } \\
n)\end{array}$ & $\begin{array}{r}\text { Stage II } \\
n)\end{array}$ & $\begin{array}{r}\text { Stage III } \\
n)\end{array}$ \\
\hline & & $47.1(8)$ & $29.4(5)$ & $23.5(4)$ \\
\hline \multirow[t]{2}{*}{$\begin{array}{l}\text { Treatment } \\
(\%, n)\end{array}$} & $\begin{array}{l}\text { Surgery/ } \\
\text { radiotherapy/ } \\
\text { chemotherapy }\end{array}$ & $\begin{array}{c}\text { Surgery/ } \\
\text { radiotherapy }\end{array}$ & $\begin{array}{c}\text { Surgery/ } \\
\text { chemotherapy }\end{array}$ & Surgery only \\
\hline & $47.1(8)$ & $11.8(2)$ & $35.3(6)$ & $5.9(1)$ \\
\hline $\begin{array}{l}\text { Currently on } \\
\text { hormone treatment } \\
(\%, n)\end{array}$ & $88.2(15)$ & & & \\
\hline $\begin{array}{l}\text { Experienced } \\
\text { menopause prior to } \\
\text { diagnosis } \\
(\%, n)\end{array}$ & $35.3(6)$ & & & \\
\hline
\end{tabular}

Post-intervention, MVPA increased to an overall mean of $17.8( \pm 13.7) \mathrm{min} / \mathrm{day}$, a mean increase of $4.5 \mathrm{~min} /$ day. Eighteen minutes represents $2.4 \%( \pm 1.5)$ of the day spent in this classification of PA. The increase of min/day of MVPA represents a smallmoderate effect $(d=0.34)$. Neither change in min/day nor time spent as a proportion of the day was statistically significant. Change in time spent as a proportion of the day in light-intensity PA $(-8.3 \%)$ and in sedentary behaviour $(7.9 \%)$, were both significantly different to baseline $(t(16)=3.522, p<0.01 ; t(16)=-3.162, p<0.01)$. The decreased time spent as a proportion of the day in light-intensity PA represents a large effect $(d=-0.85)$ and the increase in time spent in sedentary behaviour represents a moderate-large effect $(d=0.77)$. There was no significant difference in vigorous PA. 


\section{Discussion}

The trial was a 12-week intervention that aimed to increase the PA of inactive posttreatment breast cancer survivors, through using a fitness tracker combined with a behavioural counselling and goal-setting session. Post-intervention, MVPA increased by a mean of $4.5 \mathrm{~min} /$ day, a small-moderate effect $(d=0.34)$, and a magnitude of effect similar to that of other interventions for PA.[17] While only a small-moderate intervention effect, relatively small increases in PA of breast cancer survivors is associated with a reduced risk of cancer-specific and overall mortality.[18] However, the increase of MVPA is countenanced by a significant reduction in time spent in lightintensity PA and an increase in time spent sedentary. With evidence linking sedentary behaviour to an increased risk of cancer outcomes, [19] any increase of MVPA being accompanied by an increase in sedentary behaviour is a concern.

This trial is one of the first fitness tracker-based interventions delivered to inactive posttreatment breast cancer survivors. While findings are comparable with other trials conducted among cancer survivors, large standard deviations and wide confidence intervals indicate that there are apparent interindividual differences in the patterns of PA behaviour change. Therefore, it appears that only for some, fitness trackers change levels of MVPA but how effective they are, remain inconclusive.

Strengths of the trial include the $100 \%$ retention of participants and the assessment of PA by accelerometry. Previous studies have used self-report assessment which may have led to erroneous inferences being drawn on PA behaviour. The use of an objective method of assessment reduces measurement error associated with self-report estimates of PA. Use of an established, normalised equation for accelerometry data, enabled PA to be presented as estimates of the time spent in PA as a proportion of the day. In earlier work, there has been a tendency to fail to examine other classifications of PA, such as light-intensity PA and sedentary behaviour.

The sample size is the substantial limitation of this trial. Referral to the trial was by oncology staff within an oncology outpatient clinic. This approach to recruitment enabled 38 potential participants to be contacted over the recruitment period. However, 20 individuals declined to participate after being further information, and this resulted in essentially a small convenience sample. The sample is, therefore, likely subject to selection bias. The willingness of individuals to participate in a PA intervention may distinguish them from those physically inactive survivors of breast cancer who may not wish to or feel unable to change PA behaviour regardless. Potential participants may have also been dissuaded from the trial by low computer and technical literacy. Additionally, like many PA interventions, the trial was multi-component, compromising of three elements of which the fitness tracker was the primary element. Yet the efficacy of any technique included in the inherent behaviour-change techniques of the fitness tracker and associated application cannot be discerned.

Future work should attempt to identify and delineate the most effective elements of fitness tracker-based PA interventions so that only the most salient intervention components are used with specific participants. Furthermore, future work should be aware of how any increase of PA may be displaced to potentially detrimental sedentary behaviour and include methods counteract it. Use of application programming interfaces, developed and administrated by either the trial team or the fitness tracker manufacturer should also be used to allow monitoring of compliance with the intervention.

The interindividual differences in the change of patterns of physical activity behaviour suggest that only for some, fitness trackers can achieve a change in levels of 
MVPA. As any increase in MVPA appears to be accompanied by an increase in sedentary behaviour, future interventions should simultaneously aim to increase PA and reduce sedentary behaviour.

\section{References}

[1] Cormie, P., et al., The Impact of Exercise on Cancer Mortality, Recurrence, and Treatment-Related Adverse Effects. Epidemiologic Reviews, 2017. 39(1): p. 71-92.

[2] Lahart, I.M., et al., Physical activity for women with breast cancer after adjuvant therapy. The Cochrane Database of Systematic Reviews, 2018(1).

[3] Loprinzi, P.D. and H. Lee, Rationale for promoting physical activity among cancer survivors: literature review and epidemiologic examination. Oncology Nursing Forum, 2014. 41(2): p. 117-125.

[4] Blanchard, C.M., et al., A comparison of physical activity of posttreatment breast cancer survivors and noncancer controls. Behavioral Medicine, 2003. 28(4): p. 140-149.

[5] Schulmeister, L., Technology and the transformation of oncology care. Seminars in Oncology Nursing, 2016. 32(2): p. 99-109.

[6] Mercer, K., et al., Behavior Change Techniques Present in Wearable Activity Trackers: A Critical Analysis. JMIR mHealth and uHealth, 2016. 4(2): p. e40.

[7] Lynch, C., et al., Changing the Physical Activity Behavior of Adults With Fitness Trackers: A Systematic Review and Meta-Analysis. American Journal of Health Promotion, 2019. 34(4): p. 418-430.

[8] Uhm, K.E., et al., Effects of exercise intervention in breast cancer patients: is mobile health (mHealth) with pedometer more effective than conventional program using brochure? Breast Cancer Research and Treatment, 2017. 161(3): p. 443-452.

[9] Gresham, G., et al., Wearable activity monitors in oncology trials: Current use of an emerging technology. Contemporary Clinical Trials, 2018. 64: p. 13-21.

[10] Australia, E.S.S., Adult pre-exercise screening system (APSS); User Manual. 2011., Qld, Australia: ESSA.

[11] Skender, S., et al., Repeat physical activity measurement by accelerometry among colorectal cancer patients--feasibility and minimal number of days of monitoring. BMC Research Notes, 2015. 8: p. 222230.

[12] Tudor-Locke, C., et al., Utility of pedometers for assessing physical activity - Convergent validity. Sports Medicine, 2002. 32(12): p. 795-808.

[13] Troiano, R.P., et al., Physical activity in the United States measured by accelerometer. Medicine and Science in Sports and Exercise, 2008. 40(1): p. 181-188.

[14] Nguyen, N.H., et al., A qualitative evaluation of breast cancer survivors' acceptance of and preferences for consumer wearable technology activity trackers. Supportive Care in Cancer, 2017. 25(11): p. 33753384 .

[15] Lilliefors, H.W., On the Kolmogorov-Smirnov test for normality with mean and variance unknown. Journal of the American Statistical Association, 1967. 62(318): p. 399-402.

[16] Cohen, J., Statistical power analysis for the social sciences. 2nd Edition ed. 1988, Hillsdale: Lawrence Erlbaum.

[17] Bluethmann, S.M., et al., Taking the next step: a systematic review and meta-analysis of physical activity and behavior change interventions in recent post-treatment breast cancer survivors. Breast Cancer Research and Treatment, 2015. 149(2): p. 331-342.

[18] Schmid, D. and M.F. Leitzmann, Association between physical activity and mortality among breast cancer and colorectal cancer survivors: a systematic review and meta-analysis. Annals of Oncology, 2014. 25(7): p. 1293-1311.

[19] Lynch, B.M., Sedentary behavior and cancer: a systematic review of the literature and proposed biological mechanisms. Cancer Epidemiology, Biomarkers and Prevention, 2010. 19(11): p. 2691-2709. 